Effect of sheared flows on classical and neoclassical tearing modes

This article has been downloaded from IOPscience. Please scroll down to see the full text article. 2005 Nucl. Fusion 45524

(http://iopscience.iop.org/0029-5515/45/6/015)

View the table of contents for this issue, or go to the journal homepage for more

Download details:

IP Address: 122.179.52.180

The article was downloaded on 22/02/2011 at 10:30

Please note that terms and conditions apply. 


\title{
Effect of sheared flows on classical and neoclassical tearing modes
}

\author{
D. Chandra ${ }^{1}$, A. Sen ${ }^{1}$, P. Kaw ${ }^{1}$, M.P. Bora ${ }^{2}$ and S. Kruger ${ }^{3}$ \\ ${ }^{1}$ Institute for Plasma Research, Bhat, Gandhinagar 382428, India \\ ${ }^{2}$ Physics Department, Gauhati University, Guwahati 781014, India \\ ${ }^{3}$ Tech-X, Boulder, CO 8030, USA
}

Received 19 December 2004, accepted for publication 18 April 2005

Published 26 May 2005

Online at stacks.iop.org/NF/45/524

\begin{abstract}
The influence of toroidal sheared equilibrium flows on the nonlinear evolution of classical and neoclassical tearing modes is studied through numerical solutions of a set of reduced generalized MHD equations that include viscous force effects based on neoclassical closures. In general, differential flow is found to have a strong stabilizing influence leading to lower saturated island widths for the classical tearing mode and reduced growth rates for the neoclassical mode. Velocity shear, on the other hand, is seen to make a destabilizing contribution.
\end{abstract}

PACS numbers: 52.55.Fa, 52.55.Tn, 52.35.Py, 52.65.Kj

\section{Introduction}

It is now widely recognized that the $\beta$ limit of advanced tokamaks is determined by the nonlinear instabilities associated with neoclassical tearing modes (NTMs) and not by the linearized theory of ideal MHD instabilities [1-3]. In recent years, a great deal of work has been carried out on the Rutherford theory of NTMs and many important results on critical $\beta$ values and their improvement by the use of RF current drive and heating methods, stabilization by the use of external helical current coils, etc have been obtained [4-6]. There are, nevertheless, a number of issues related to the origin of excitation of the mode, its excitation threshold, its nonlinear behaviour and its interaction with error fields and equilibrium shear flows that have not been satisfactorily resolved and need to be better understood [7]. The influence of shear flows is a particularly important issue since sheared velocity flows are known to be widely prevalent in tokamak devices and can be generated by neutral beams, ion cyclotron heating and self-consistent drift turbulence. A number of past studies have examined the effect of flows on tearing modes, particularly in the linear regime and for simplified geometries [8]. There have also been a few nonlinear studies $[9,10]$ but the problem is quite complex, particularly in realistic toroidal geometries, and is an important area of present and future study for major numerical initiatives such as NIMROD [11]. In this paper, we report on numerical studies that we have carried out on this problem with the help of a finite difference code NEAR that solves a set of generalized reduced MHD equations [12] and that includes viscous force effects based on neoclassical closures. The code has been tested in the past for the effect of flows on linear resistive tearing modes. Our emphasis in this work is to extend the investigation to the nonlinear regime for both classical tearing modes and
NTMs and to delineate the physical mechanisms that govern the influence of flow on the evolution of the modes.

\section{Model equations}

Our numerical simulations are based on the solutions of a set of reduced MHD equations originally proposed by Kruger et al in 1998 [12]. These equations, which are valid at any aspect ratio, are derived using $k_{\|} / k_{\perp}$ (the ratio of parallel to perpendicular wave numbers) as a small expansion parameter and by employing a multiple scale analysis that respects equilibrium constraints and also permits elimination of fast time scales associated with perpendicular wave motion. The model equations thus evolve scalar potential quantities on a time scale associated with the parallel wave vector (shearAlfvén wave time scale), which is the time scale of interest for resistive MHD instabilities like tearing modes. In addition, the model equations also permit the incorporation of subAlfvénic equilibrium shear flows and neoclassical closures in a straightforward manner and are therefore well suited for studying the nonlinear evolution of NTMs in the presence of flows. In the limit of $\beta \sim \delta^{1 / 2}(\delta \ll 1)$, a simplified set of the evolution equations are as follows:

$$
\begin{aligned}
& \frac{\partial \Psi}{\partial t}-\left(\boldsymbol{b}_{0}+\boldsymbol{b}_{1}\right) \cdot \nabla \phi_{1}-\boldsymbol{b}_{1} \cdot \nabla \phi_{0}=\eta \tilde{J}_{\|}-\frac{1}{n e} \boldsymbol{b}_{0} \cdot \nabla \cdot \boldsymbol{\Pi}_{\mathrm{e}} \\
& \nabla \cdot\left(\frac{\rho}{B_{0}} \frac{\mathrm{d}}{\mathrm{d} t} \frac{\nabla \phi_{1}}{B_{0}}\right)+\left(\boldsymbol{V}_{1} \cdot \nabla\right)\left(\nabla \cdot\left(\frac{\rho}{B_{0}} \frac{\nabla \phi_{0}}{B_{0}}\right)\right) \\
& =\left(\boldsymbol{B}_{0} \cdot \nabla\right) \frac{\tilde{J}_{\|}}{B_{0}}+\left(\boldsymbol{B}_{1} \cdot \nabla\right) \frac{J_{T \|}}{B_{0}}+\nabla \cdot \frac{\boldsymbol{B}_{0} \times \nabla p_{1}}{B_{0}^{2}} \\
& \quad+\nabla \cdot \frac{\boldsymbol{B}_{0}}{B_{0}^{2}} \times \nabla \cdot \boldsymbol{\Pi}
\end{aligned}
$$


Effect of sheared flows on classical and NTMs

$$
\begin{aligned}
& \frac{\mathrm{d} p_{1}}{\mathrm{~d} t}+\left(\boldsymbol{V}_{1} \cdot \nabla\right) p_{0}+\Gamma p_{\mathrm{T}} \nabla \cdot \boldsymbol{V}_{1} \\
& \quad=(\Gamma-1)\left[\eta J_{\mathrm{T} \|}^{2}-\boldsymbol{\Pi}: \nabla \boldsymbol{V}+\boldsymbol{\Pi}_{\mathrm{e}}: \nabla \frac{\boldsymbol{J}}{n e}-\nabla \cdot \boldsymbol{q}\right] \\
& \rho \frac{\mathrm{d} \tilde{V}_{\|}}{\mathrm{d} t}+\left(\boldsymbol{V}_{1} \cdot \nabla\right) V_{\|_{0}}=-\boldsymbol{b}_{0} \cdot \nabla p_{1}-\boldsymbol{b}_{1} \cdot \nabla p_{\mathrm{T}}-\boldsymbol{b}_{0} \cdot \nabla \cdot \boldsymbol{\Pi} \\
& \nabla \cdot \boldsymbol{q}=-\chi_{\perp} \nabla^{2} p_{1}-\left(\chi_{\|}-\chi_{\perp}\right)\left[\boldsymbol{b}_{1} \cdot \nabla\left(\boldsymbol{b}_{0} \cdot \nabla p_{0}\right)\right. \\
& \quad+\boldsymbol{b}_{0} \cdot \nabla\left(\boldsymbol{b}_{0} \cdot \nabla p_{1}+\boldsymbol{b}_{1} \cdot \nabla p_{0}\right)+\boldsymbol{b}_{0} \cdot \nabla\left(\boldsymbol{b}_{1} \cdot \nabla p_{1}\right) \\
& \left.\quad+\boldsymbol{b}_{1} \cdot \nabla\left(\boldsymbol{b}_{1} \cdot \nabla p_{0}\right)+\boldsymbol{b}_{1} \cdot \nabla\left(\boldsymbol{b}_{0} \cdot \nabla p_{1}\right)\right]
\end{aligned}
$$

where

$$
\begin{aligned}
& \frac{\mathrm{d}}{\mathrm{d} t}=\frac{\partial}{\partial t}+\boldsymbol{V} \cdot \nabla ; \quad \Phi_{\mathrm{T}}=\Phi_{0}+\Phi_{1} ; \quad p_{\mathrm{T}}=p_{0}+p_{1} ; \\
& \boldsymbol{b}_{\mathrm{T}}=\boldsymbol{b}_{0}+\boldsymbol{b}_{1}=\frac{\boldsymbol{B}_{0}}{B_{0}}+\frac{\boldsymbol{B}_{1}}{B_{0}}, \\
& J_{\mathrm{T} \|}=J_{0 \|}+\tilde{J}_{\|}=\boldsymbol{b}_{0} \cdot \nabla \times \boldsymbol{B}_{0}+\nabla^{2} \Psi, \\
& \boldsymbol{V}=\Omega(\psi) R^{2} \nabla \zeta+\boldsymbol{V}_{1}=\frac{\boldsymbol{B}_{0} \times \nabla \Phi_{0}}{B_{0}^{2}}+V_{0 \|} \boldsymbol{b}_{0} \\
& +\frac{\boldsymbol{B}_{0} \times \nabla \Phi_{1}}{B_{0}^{2}}+\tilde{V}_{\|} \boldsymbol{b}_{\mathrm{T}} .
\end{aligned}
$$

Equation (1) is Ohm's law, with $\Psi$ representing the magnetic flux, $\phi$ the electrostatic potential, $J$ the current density, $\eta$ the resistivity, $B$ the magnetic field, $n$ the number density and $\Pi_{\mathrm{e}}$ the electron stress tensor. The last term on the right-hand side is the contribution from the bootstrap current and is the driving term for the NTM. Equation (2) is the vorticity equation with $\boldsymbol{V}$ the flow velocity, $\rho$ the mass density and $\boldsymbol{\Pi}$ the total stress tensor. Equation (3) is the pressure evolution equation where heat flow terms have been retained with $\boldsymbol{q}$ representing the heat flux and $\chi_{\perp}, \chi_{\|}$representing the heat transport coefficients in the perpendicular and parallel directions, respectively. Finally, equation (4) is the evolution equation for the parallel velocity component. The subscripts 0,1 and $\mathrm{T}$ refer to equilibrium, perturbed and total quantities, respectively. The equilibrium toroidal velocity, which is conveniently expressed in terms of a function $\Omega(\psi)$, is ordered such that $V_{0} / V_{\mathrm{A}} \sim \epsilon \ll 1$ so that the flows are restricted to the sub-Alfvénic range. The above equations have been programmed into an initial value code, called NEAR, which is a derivative of an older code called FAR. An early benchmarking of this code was carried out in [13], where terms proportional to $\Pi_{\mathrm{e}}, \boldsymbol{\Pi}$ and $\nabla \cdot \boldsymbol{q}$ were dropped and the tests were restricted to the linear growth regime of classical tearing modes. Our emphasis, in this work, is to explore the effects of shear flow in the nonlinear regime and in particular to examine its influence on the evolution of NTMs.

\section{Numerical simulation results}

\subsection{Classical tearing modes}

As a preliminary to our study of the NTMs and for proper benchmarking of the code in the nonlinear regime we have first studied the nonlinear evolution of the classical tearing mode and the effect of sheared flow on its evolution. For this we have adopted the simplified approach of [13] and dropped the stress tensor contributions as well as the $\nabla \cdot \boldsymbol{q}$ term in the model. In the absence of flow, we

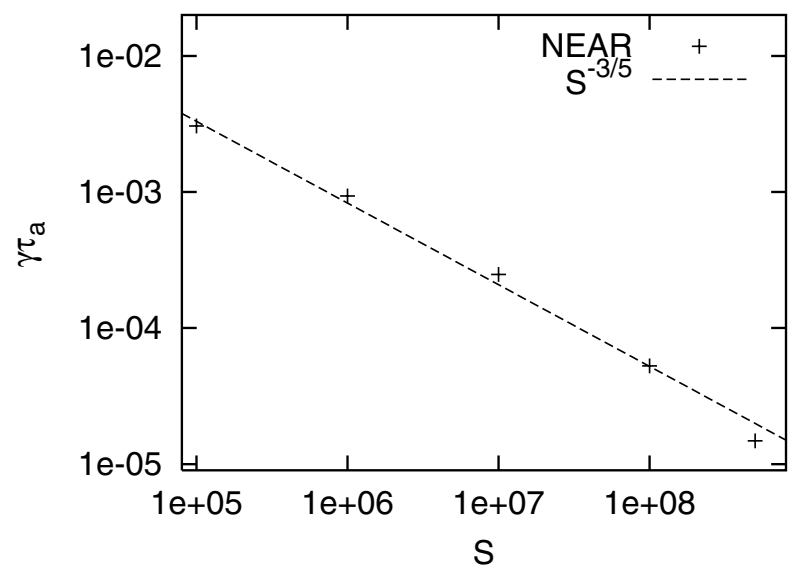

Figure 1. Benchmark results confirming the $S^{-3 / 5}$ scaling of the linear growth rate of the $(m / n=2 / 1)$ classical tearing mode.

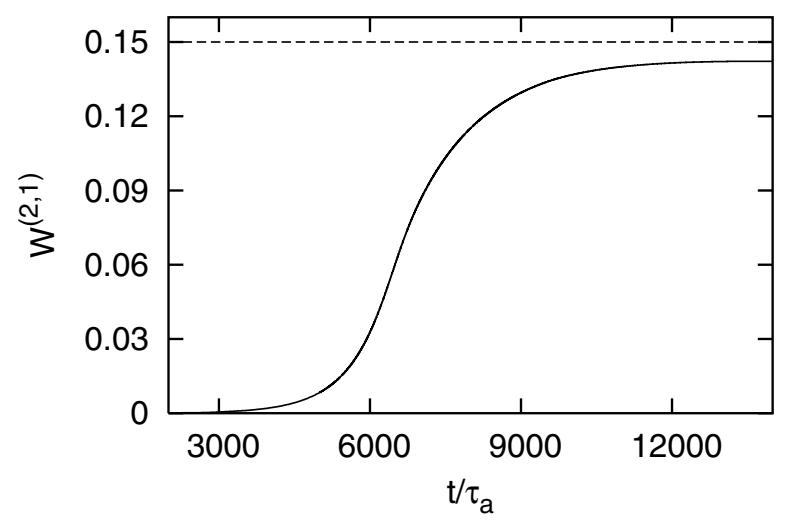

Figure 2. Nonlinear saturation of the $(m / n=2 / 1)$ classical tearing mode.

generate an equilibrium profile by numerically solving the Grad-Shafranov equation with the help of an equilibrium code called TOQ (http://fusion.gat.com/toq). Using this equilibrium in NEAR we study the evolution of the $(m / n=$ $2 / 1$ ) tearing mode from an initial arbitrary perturbation. In the linear regime the growth rate of the $(2 / 1)$ mode displays all the characteristic scalings of the resistive tearing instability ( viz. $\gamma \sim S^{-3 / 5} \Delta^{\prime 4 / 5}$ etc, where $S$ is the Reynolds number and $\Delta^{\prime}$ the outer region mismatch parameter) in agreement with earlier benchmark results [13]. Figure 1 illustrates one such benchmark test showing the scaling of the linear growth rate with $S$. In the nonlinear regime the growth rate slows down to the algebraic rate of the Rutherford regime and eventually saturates with an island width $W_{\text {sat }}$ that is in good agreement with theoretical estimates based on the nonlinear modification of $\Delta^{\prime} \rightarrow \Delta^{\prime}\left(1-W / W_{\text {sat }}\right)$ [14]. This is shown in figure 2 where the dashed line corresponds to the theoretical saturated island width. We next incorporate sheared flow in the system by generating an appropriate flow modified equilibrium from TOQ and using it in NEAR.

For our numerical studies, we have used two kinds of flow profiles to identify effects arising from differential flow and flow shear. The typical flow profiles are shown in figure 3, where profile 1 has only differential flow between two adjacent mode rational surfaces $((2,1)$ and $(3,1)$ in this instance $)$ and no shear, whereas profile 2 has both differential flow and 


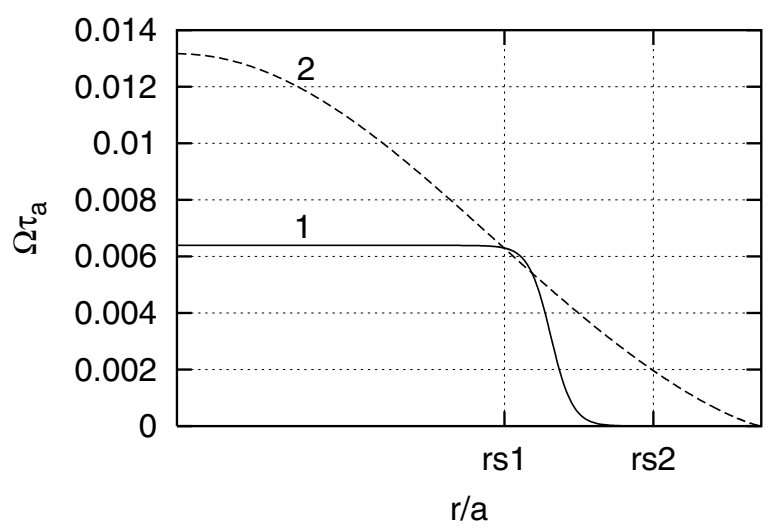

Figure 3. Typical equilibrium toroidal flow profiles: 1, differential flow with no shear at the mode rational surfaces; 2 , differential flow with finite shear at the mode rational surfaces.

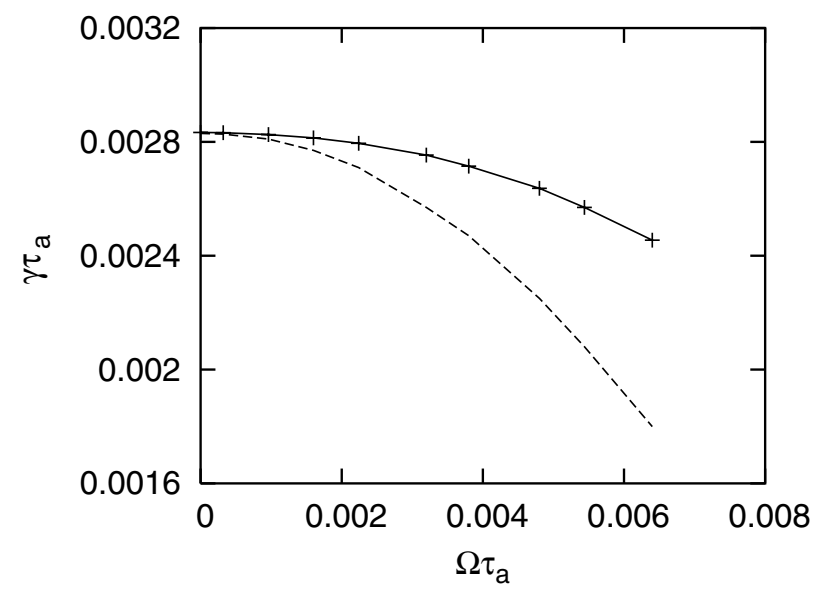

Figure 4. The reduction in growth rate of the $(m / n=2 / 1)$ classical tearing mode as a function of the differential flow amount. The lower curve is from the analytic solution of the model dispersion relation (7).

shear. In the linear regime, differential flow is found to have a stabilizing effect, as illustrated in figure 4 where the growth rate is seen to decrease with increasing differential flow. On the other hand, inclusion of shear in the velocity (e.g. the use of profile 2 for the equilibrium profile) diminishes the stabilizing effect. To understand the origins of the stabilizing and destabilizing influences we have carried out detailed controlled numerical runs with particular terms of the code (corresponding to various physical contributions) succesively turned on and off. In the absence of flow, it is well known that the pressure-curvature term has a stabilizing influencethe so-called Glasser-Greene-Johnson effect [15]. Another important physical effect arises out of toroidal coupling of the various mode resonant surfaces. We have examined the contributions of these effects in NEAR by artificially turning this term off and comparing the values of the linear growth rate in each case. Table 1 summarizes a set of typical results. As can be seen, the pressure-curvature term has a stabilizing influence and its stabilizing contribution is further enhanced in the presence of differential flow. The enhancement can be attributed to the centrifugal force modification of the equilibrium pressure surfaces. Toroidal coupling is also found to have a stabilizing influence and this is also enhanced by
Table 1. Summary of quantitative contributions of various linear terms in NEAR (corresponding to different physical effects) on the linear growth rate of classical tearing modes.

\begin{tabular}{llll}
\hline $\begin{array}{l}\text { Toroidal } \\
\text { coupling }\end{array}$ & $\begin{array}{l}\text { Pressure- } \\
\text { curvature }\end{array}$ & $\begin{array}{l}\text { Equilibrium } \\
\text { flow }\end{array}$ & $\begin{array}{l}\text { Linear } \\
\text { growth rate }\end{array}$ \\
\hline Off & Off & Off & $2.90 \mathrm{e}-3$ \\
Off & On & Off & $2.85 \mathrm{e}-3$ \\
On & Off & Off & $2.20 \mathrm{e}-3$ \\
On & On & Off & $2.01 \mathrm{e}-3$ \\
Off & Off & On & $2.84 \mathrm{e}-3$ \\
Off & On & On & $2.80 \mathrm{e}-3$ \\
On & Off & On & $2.11 \mathrm{e}-3$ \\
On & On & On & $1.89 \mathrm{e}-3$ \\
\hline
\end{tabular}

differential flow. To understand this mechanism, we consider a simple analytic model that is a generalization of the single mode tearing mode theory and captures the essential physics of mode coupling. When different mode rational surfaces are toroidally coupled, the eigenfrequencies of the system are determined from a generalized dispersion relation based on a matching of a $\Delta^{\prime}$ matrix (the outer response matrix) [16,17] to an inner layer matrix response $\Delta(\omega)$. For the simple case of two coupled rational surfaces (e.g. $(2,1)$ and $(3,1))$, the linear dispersion relation can be written in the form

$$
\operatorname{det}\left[\begin{array}{cc}
\Delta_{11}^{\prime}-\Delta_{11}(\omega) & \Delta_{12}^{\prime} \\
\Delta_{21}^{\prime} & \Delta_{22}^{\prime}-\Delta_{22}(\omega)
\end{array}\right]=0,
$$

where $\Delta_{i j}(\omega)=-i\left(\omega-\Omega_{j}\right) \tau_{L j} \delta_{i j}$, with $\Omega_{j}$ the equilibrium flow frequency at the surface $j$ and $\tau_{L j}$ is a parameter that describes the inner layer response and is a function of the resistivity, viscosity and the inertia at the mode rational surface $j$. This yields a quadratic equation for $\omega$,

$$
\begin{gathered}
\omega^{2}-\omega\left[\Omega_{1}+\Omega_{2}+i\left(\gamma_{11}+\gamma_{22}\right)\right]+\frac{\left[\Omega_{1}+\Omega_{2}+i\left(\gamma_{11}+\gamma_{22}\right)\right]^{2}}{4} \\
+\gamma_{12} \gamma_{21}-\frac{\left[\Delta \Omega+i\left(\gamma_{22}-\gamma_{11}\right)\right]^{2}}{4}=0,
\end{gathered}
$$

where $\gamma_{i i}=\Delta_{i i}^{\prime} / \tau_{L i}$ are the individual growth rates at the surfaces $i$ for the uncoupled system, $\gamma_{i j}=\Delta_{i j}^{\prime} / \tau_{L i}$ are the coupling coefficients, $\Omega_{i}$ is the equilibrium flow frequency at the surface $i$ and $\Delta \Omega=\Omega_{2}-\Omega_{1}$ is the amount of differential flow. Using NEAR we have determined $\gamma_{i i}$ for the two individual surfaces by turning off the toroidal coupling and the flow. Using these values and the value of $\omega$ obtained in the presence of coupling we have solved equation (7) to obtain the value of $\gamma_{12} \gamma_{21}$. Using these values of $\gamma_{i j}$, equation (7) is subsequently solved for $\omega$ at various values of $\Omega_{1}$ and $\Omega_{2}$. The results show a decrease in the growth rate of the mode as $\Delta \Omega$ increases in agreement with the trend shown by the results of NEAR in the presence of flow. Figure 4 shows a comparison of the results of NEAR with that of the simple analytic model.

Physically, differential flow acts to decouple the surface, so that at a given rational surface the other rational surface appears as a conducting surface and provides a stabilizing effect. To state it differently, the amount of reconnection at a given surface decreases as a result of differential rotation. This can also be ascertained qualitatively from the simple analytic model discussed earlier. Taking the surface 1 to correspond to the $(3,1)$ resonant surface and surface 2 to correspond to the $(2,1)$ mode, one can show that the ratio of the eigenvectors is 


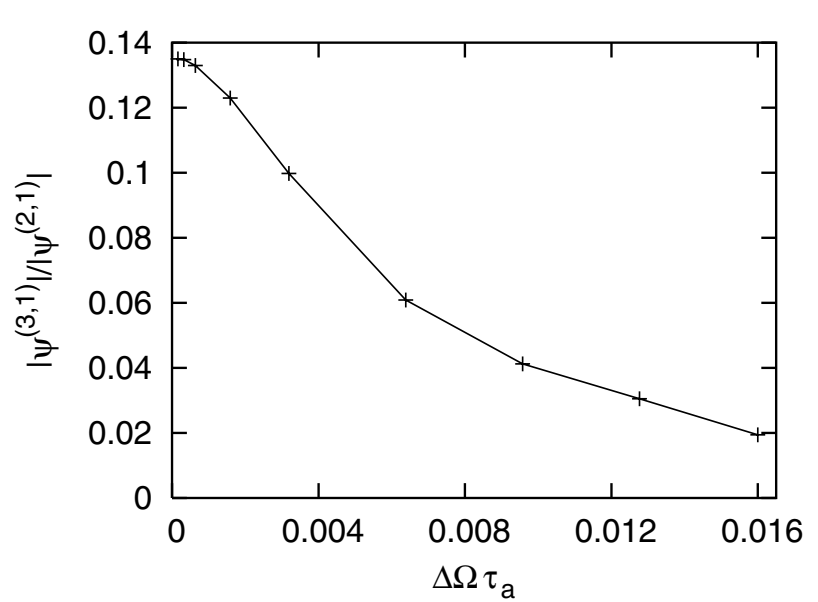

Figure 5. Decrease in reconnection at the $(3,1)$ surface with increasing differential rotation.

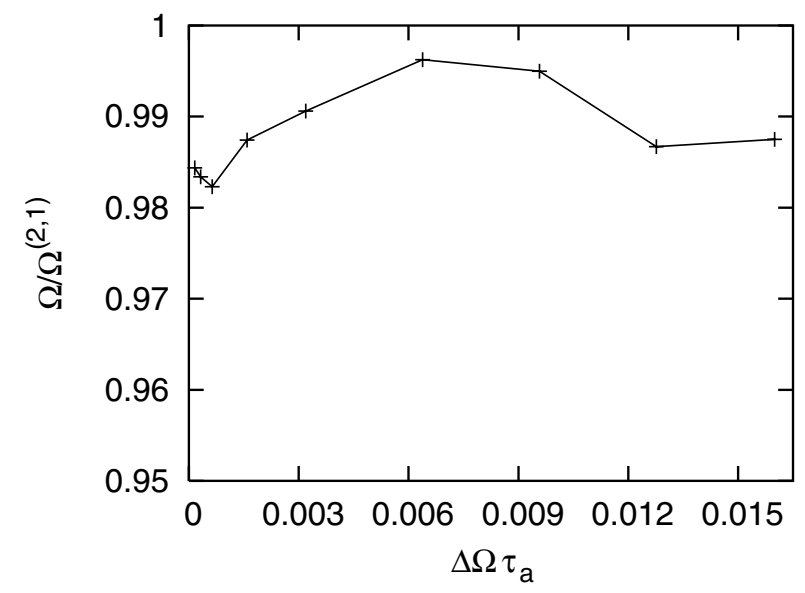

Figure 6. Ratio of $(2,1)$ classical mode real frequency to flow rotation frequency as a function of differential flow.

given as

$$
\frac{\left|\Psi_{3,1}\right|}{\left|\Psi_{2,1}\right|} \approx \frac{\gamma_{1,2}}{\Delta \Omega}
$$

Thus, the amount of reconnection at $(3,1)$ decreases with increasing $\Delta \Omega$. In figure 5 we have plotted this ratio as obtained from NEAR and we note that this trend is borne out. The other effect of differential rotation is to make the mode rotate at a velocity close to the equilibrium rotation frequency at the mode rational frequency. Such an effect is also confirmed in NEAR as seen from figure 6 , where the mode rotation frequency is plotted for various values of $\Omega$. So far, we have ignored the effect of flow shear, which, as stated earlier, has a destabilizing effect. This can be understood from flow induced changes in the inner layer dynamics, as has been extensively worked out in [8]. For the low values of flow shear we have used, the approximate growth rate of the linear tearing mode is given by [8]

$$
\gamma \sim \alpha^{2 / 5} \Delta^{\prime 4 / 5} S^{-3 / 5} \hat{\gamma}
$$

where $\alpha$ is the normalized wave number and $\hat{\gamma} \geqslant 1$ is the flow shear correction factor. The results from NEAR for our parameter range agree with this trend.

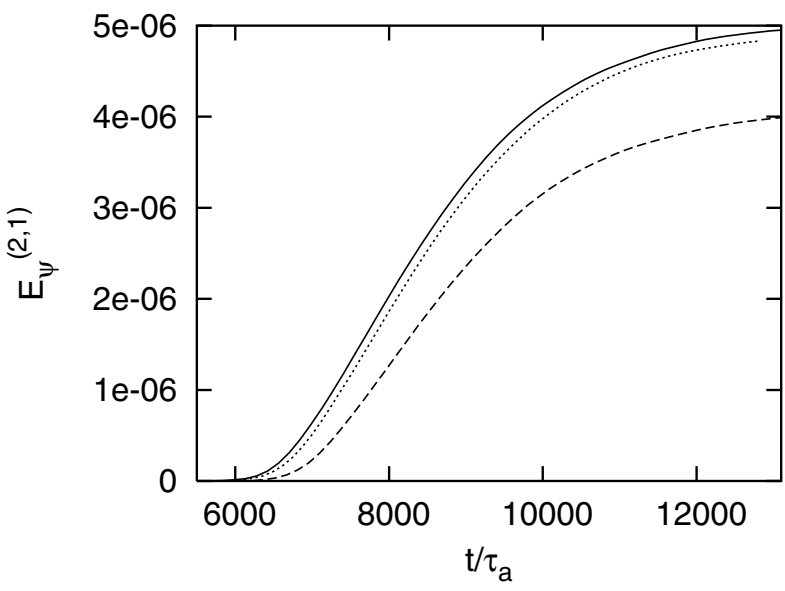

Figure 7. Evolution of mode energy for the $(m / n=2 / 1)$ classical mode for the no flow case (top curve), flow profile 1 (bottom curve) and flow profile 2 (middle curve).

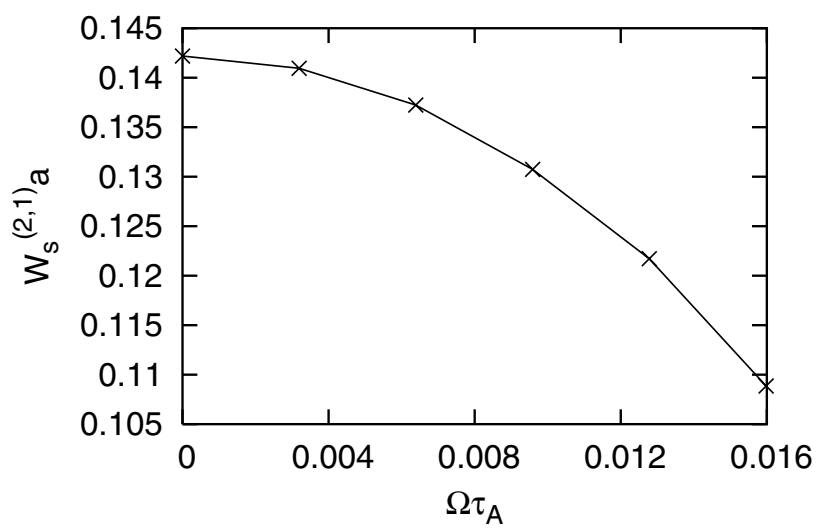

Figure 8. Decrease of the saturated island width for the classical $(2,1)$ mode as a function of the differential flow amount.

In the nonlinear regime, the differential flow effect continues to have a stabilizing influence and leads to a lower level of mode saturation as shown by the lowest curve of figure 7. The mode rotation frequency is close to the flow frequency but shows some nonlinear increase close to the saturation region. When we use profile 2 we find a decrease in the stabilization effect (the intermediate curve in figure 7) indicating that velocity shear has a destabilizing trend. The saturated island width decreases with the amount of differential flow as shown in figure 8 . Since an analytic model of the nonlinear characteristics of the mode does not exist, the insights gained from the linear behaviour do provide a useful guideline and we observe the predominance of the same physical effects in the nonlinear regime. It should be mentioned, though, that there may be other effects associated with parallel velocity coupling and global changes affecting $\Delta^{\prime}$, which, though not important for our present choice of flow values, may play an important contributory role in other parametric regimes of flow velocity and flow shear.

\subsection{Neoclassical tearing modes}

As is well known, the NTM is driven unstable by a perturbation of the bootstrap current, which arises from the viscous damping 
of the poloidal electron flow and which is proportional to the cross-field pressure gradient. In the presence of a seed magnetic island, the equilibrium pressure gradient flattens locally, thereby switching off the bootstrap current; this results in a negative current perturbation on the given rational surface, which drives up the amplitude of the magnetic island by the Rutherford nonlinear growth mechanism. The pressure flattening relies on the assumption that the parallel transport is much faster than the perpendicular transport. To study the evolution of NTMs, it is, therefore, necessary to retain the stress tensor terms in the reduced MHD equations in order to provide the drive term and to keep the heat flow terms in the pressure evolution equation. For the neoclassical viscous stress tensor we have used the following closure ansatz [12]:

$$
\nabla \cdot \vec{\Pi}_{j}=\rho_{j} \mu_{j}\left\langle B^{2}\right\rangle \frac{\boldsymbol{V}_{j} \cdot \nabla \theta}{(\boldsymbol{B} \cdot \nabla \theta)^{2}} \nabla \theta
$$

where $j=i, e$ and $\mu_{j}$ is the viscous damping frequency of each species $j$. This closure ansatz is appropriate for the long mean free path (low collisional) limit and reproduces poloidal flow damping as well as gives an appropriate bootstrap current perturbation.

The NTM instability is essentially a nonlinear (or subcritical) instability that can develop even in the limit of resistive MHD stability (i.e. $\Delta^{\prime}<0$ ) and requires a threshold amplitude of the magnetic perturbation for its excitation. The evolution of a single helicity NTM is well described by the Rutherford nonlinear theory, suitably modified to include neoclassical effects [18-21]. A generalized form of the Rutherford equation is given by

$$
\begin{aligned}
& \frac{\tau_{\mathrm{R}}}{r_{\mathrm{s}}} \frac{\mathrm{d} W}{\mathrm{~d} t}=r_{\mathrm{s}} \Delta^{\prime}+r_{\mathrm{s}} \beta_{\mathrm{p}}\left(-\frac{a_{\mathrm{GGJ}}}{\sqrt{W^{2}+0.2 W_{\mathrm{d}}^{2}}}+a_{\mathrm{bs}} \frac{W}{W^{2}+W_{\mathrm{d}}^{2}}\right. \\
& \left.-\frac{a_{\mathrm{pol}}}{W^{3}} h(W)\right),
\end{aligned}
$$

where $\tau_{\mathrm{R}}$ is the resistive time at the resonant flux surface located at the radius $r=r_{\mathrm{s}}$ and $\beta_{\mathrm{p}}$ is the poloidal beta. The first term on the RHS of the above equation (11) is the usual driving term for tearing modes and is positive for resistive MHD instability. For the case of an NTM, as discussed earlier, we take this term to be negative. The next term proportional to $a_{\mathrm{GGJ}}$ is the stabilizing contribution of the curvature due to toroidicity, the Glasser-Greene-Johnson effect, which has been discussed in the previous section in the context of classical tearing modes. Finite perpendicular heat diffusion modifies this term over the scale length [22] $W_{\mathrm{d}}=5.1 r_{\mathrm{s}}\left(\chi_{\perp} / \chi_{\|}\right)^{0.25}\left[R /\left(r_{\mathrm{s}} s_{\mathrm{s}} n\right)\right]^{0.5}$, where $\chi_{\|}$and $\chi_{\perp}$ are the parallel and perpendicular pressure diffusivities, respectively, $R$ is the major radius, $n$ the toroidal mode number and $s_{\mathrm{s}}$ the magnetic shear at the resonant surface. The third term is the destabilizing contribution of the perturbed bootstrap current. The sign of $a_{\mathrm{bs}}$ depends on the relative signs of the pressure gradient and magnetic shear (it is positive for a normal profile where the pressure gradient is negative and the magnetic shear is positive and is negative in a reversed shear region). The final term is the ion polarization current contribution and its sign depends on the island rotation frequency with respect to the electron and ion diamagnetic frequencies. However, it should be noted that this term arises in a two fluid model and this effect is absent in the one fluid

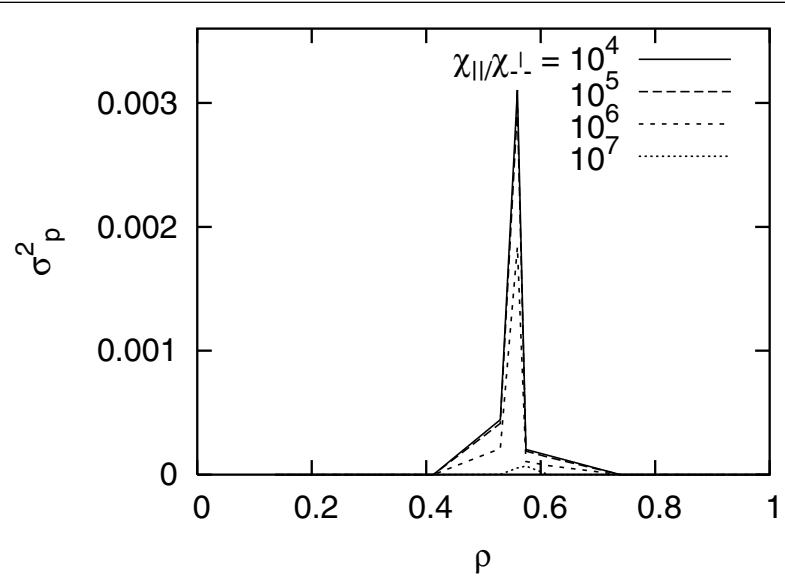

Figure 9. The pressure variance $\sigma_{\mathrm{p}}^{2}$, computed by following individual field lines as a function of $\chi_{\|} / \chi_{\perp}$. It shows that the degree of pressure equilibration increases with increase in the ratio $\chi_{\|} / \chi_{\perp}$.

reduced MHD description of NEAR. We will, therefore, not discuss this effect in our work.

From equation (11) it is clear that for an NTM instability to occur the contribution of the third term must be larger than the sum of the first two terms. In other words, there exists a threshold amplitude in $W$ for the instability to be triggered, which is a characteristic signature that distinguishes it from the classical tearing mode. For a given value of a perturbation, one can also similarly determine a threshold in $\beta$. Before investigating the effect of flows on NTMs we have benchmarked the NEAR code by reproducing these characteristic features of the NTMs and also paid particular attention to pressure equilibration [23].

Figure 9 shows the necessary pressure equilibration, which is key to NTM physics. The typical ratio of $\chi_{\|} / \chi_{\perp}$ in most of our runs has been of the order of $10^{6}$ or more and the Reynolds number $S$ has been kept at $10^{5}$ or higher. The amount of viscosity chosen is such that the ratio of resistive time to viscous time is about $10^{-1}-10^{-2}$. We have studied the evolution of the $(m / n=2 / 1)$ and $(m / n=3 / 1)$ NTM modes using equilibrium configurations for which the corresponding classical modes are linearly stable. In figure 10, we show the dependence of the mode evolution on the initial amplitude and the existence of a threshold amplitude for the destabilization of the mode. In figure 11, we have demonstrated the existence of the threshold $\beta$ above which the mode gets unstable. Figure 12 shows a typical nonlinear evolution of the island width and its eventual saturation. The magnetic flux function profile and contour showing islands for the typical evolution are shown in figures 13 and 14 , respectively.

We next introduce toroidal flow in the system through equilibrium flow profiles of types 1 and 2 (cf figure 3 ) by generating them self-consistently with TOQ. The basic trend in this case is seen to be quite similar to what we observe for classical tearing modes, namely destabilizing effects of flow shear and a more dominant stabilizing effect from differential flow. In figure 15 we show the mode energy evolution for three different cases - the top curve is without any flow, the bottom curve is for a flow profile of type 1 (pure differential flow) and the intermediate curve is for a flow profile of type 2 (differential flow + shear). The qualitative behaviour in the nonlinear 


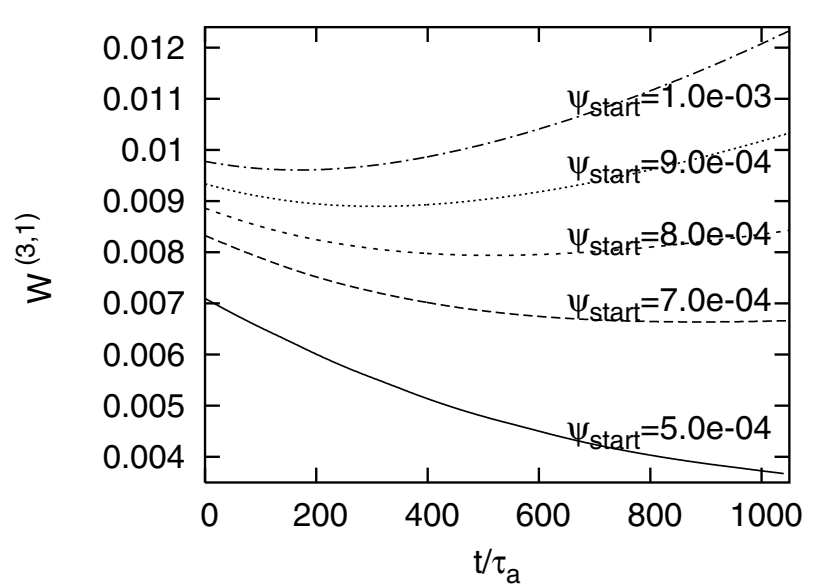

Figure 10. Benchmark results showing the existence of a threshold amplitude for the $(3,1)$ NTM.

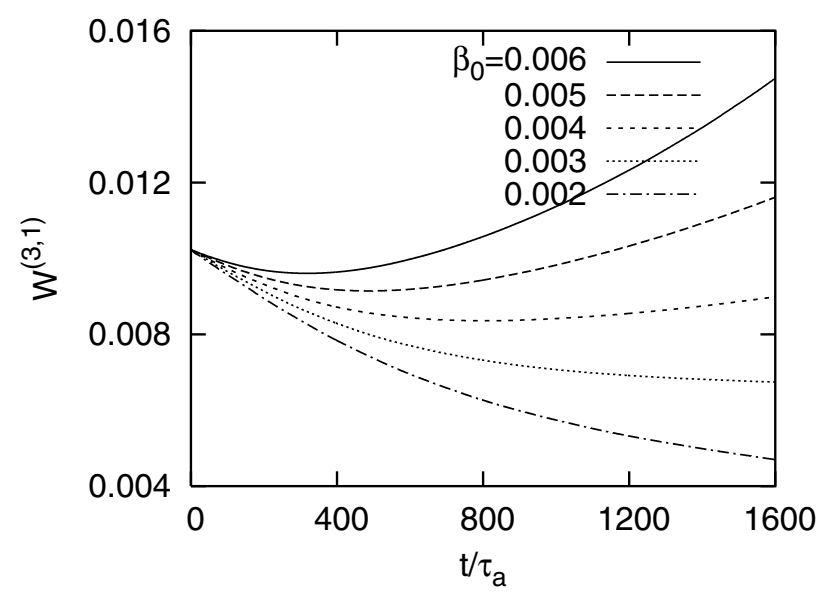

Figure 11. Benchmark results showing the existence of a threshold $\beta$ for a given initial perturbation in the case of a $(3,1)$ NTM.

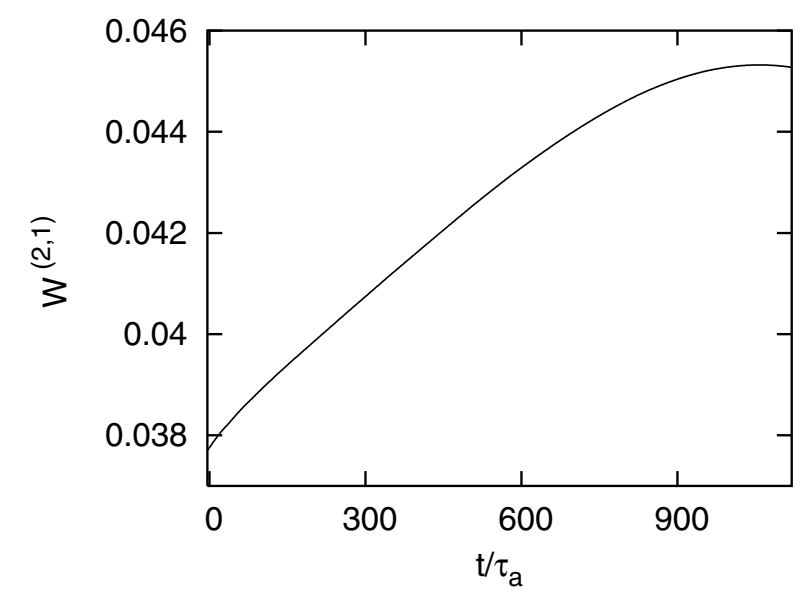

Figure 12. Benchmark result showing typical island width of the $(2,1)$ NTM and its evolution to saturation.

regime is once again found to be quite similar to that observed for classical tearing modes discussed in the previous section. However, there is a quantitative difference in the magnitude of stabilization for the NTM case (figure 15 compared with figure 7) primarily due to the difference in the magnitude of

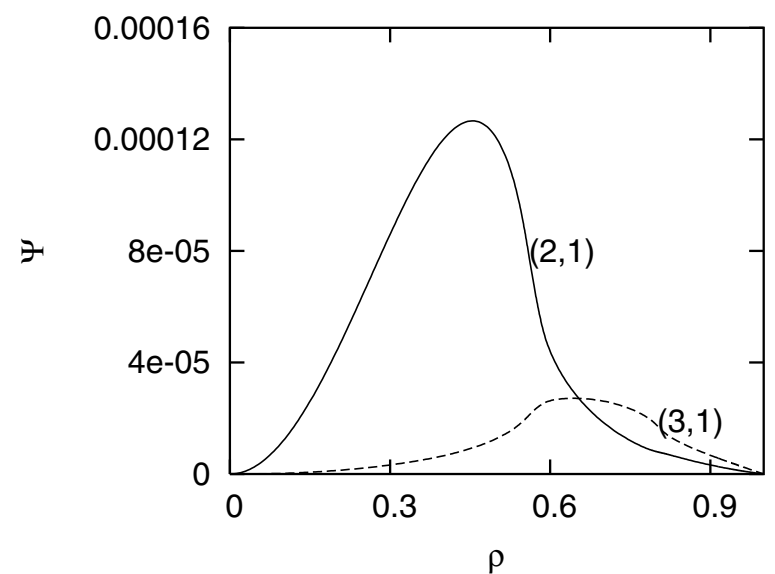

Figure 13. Benchmark result showing the profiles of the magnetic flux function $\Psi$ for the $(2,1)$ and $(3,1)$ modes for a typical NTM evolution.

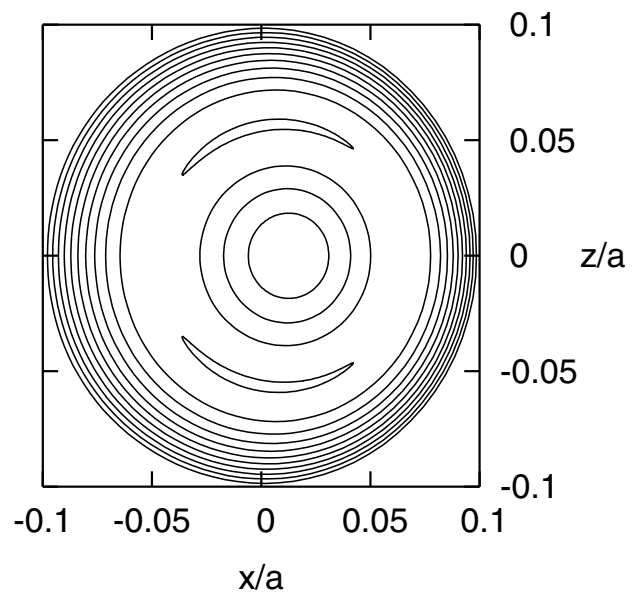

Figure 14. Benchmark result: the helical contour of the magnetic flux function $\Psi$ for a typical NTM evolution showing the formation of a $(2,1)$ magnetic island.

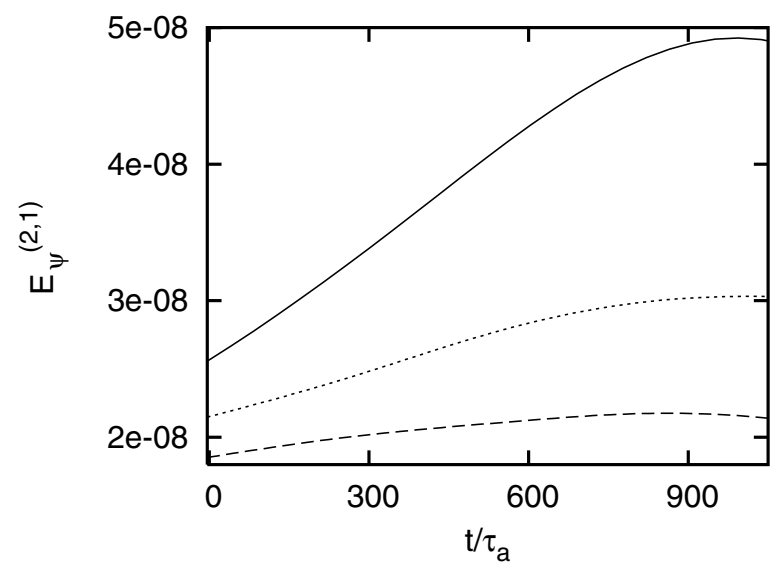

Figure 15. Nonlinear evolution of the $(2,1)$ NTM for no flow (top curve), flow profile-1 (bottom curve) and flow profile-2 (middle curve).

the differential flow for the two cases. This happens because in generating the equilibrium flow profiles in TOQ we have kept the magnitude of the Mach number parameter $\left(V_{0} / C_{\mathrm{s}}\right)$ the same in both the cases but the plasma $\beta$ values have been 
chosen to be different. The NTM runs have been made at a higher $\beta$ value compared with the classical ones in order to be well beyond the threshold regime. Thus the magnitude of $\Omega \tau_{\mathrm{a}}=V_{0} / V_{\mathrm{A}}=\beta V_{0} / C_{\mathrm{s}}$ at the $(2,1)$ surface is higher for the NTM runs $(\sim 0.0095)$ as compared with the classical case ( $\sim 0.0064$ as shown in figure 3$)$ and consequently leads to a larger amount of stabilization. If the Mach number parameter is chosen differently in the two cases, so as to keep the differential flow amount the same, then the stabilization effects are found to be of comparable magnitude.

\section{Summary and discussion}

Our present set of numerical results, using two different profiles of toroidal equilibrium flow, indicates that differential flow has a strong stabilizing influence on the nonlinear evolution of both classical and NTMs whereas velocity shear has a destabilizing effect. While a quantitative comparison with any existing analytic model is not possible, some qualitative features of the results can be understood on the basis of past theoretical work on shear flows, as well as nonlinear evolutionary studies of tearing modes in the absence of flows. The destabilization effect of weak shear flows is consistent with the findings of earlier linear studies [8].

The source of the stabilizing influence of differential flow has been primarily traced to two physical factors- the pressure-curvature term and the toroidal coupling. The pressure-curvature term has a stabilizing influence (when the overall curvature is favourable) even in the absence of flow. We have confirmed this by artificially turning this term off in the code. We have further numerically confirmed that the stabilizing effect of this term is enhanced by the presence of differential flow due to equilibrium modifications of the pressure profile caused by the centrifugal effects of flow. However, the measured enhancement is not sufficient to fully account for the amount of flow induced stabilization. The additional stabilization comes about from the influence of differential flow on toroidal mode coupling. A semiquantitative measure of this effect is obtained by a simple model application of the $\Delta^{\prime}$ matrix theory and the results are seen to agree with the numerical findings quite well in the linear regime. Flow induced changes observed in the linear regime are found to continue in the nonlinear regime where differential flows lead to lower island saturation levels whereas flow shear appears to oppose this stabilizing trend. Further explorations with different flow profiles and different neoclassical closure models are currently in progress to study their influence on the nonlinear evolution of NTMs.

\section{References}

[1] Hegna C.C. 1998 Phys. Plasmas 51767

[2] Chang Z. et al 1995 Phys. Rev. Lett. 744663

[3] Carrera R., Hazeltine R.D. and Kotschenreuther M. 1986 Phys. Fluids 29899

[4] Rutherford P.H. 1973 Phys. Fluids 141903

[5] Zohm H. et al 1999 Nucl. Fusion 39577

[6] Yu Q., Gunter S. and Lackner K. 2004 Phys. Plasmas 11140

[7] ITER Physics Expert Group on Disruptions, Plasma Control and MHD, ITER Physics Basis (ed) 1999 Nucl. Fusion 39 2251

[8] Chen X.L. and Morrison P.J. 1990 Phys. Fluids B 2495

[9] Persson M. and Bondeson A. 1990 Phys. Fluids B 22315

[10] Chen X.L. and Morrison P.J. 1992 Phys. Fluids B 4845

[11] Glasser A.H. et al 1999 Plasma Phys. Control. Fusion 41 A747

[12] Kruger S.E., Hegna C.C. and Callen J.D. 1998 Phys. Plasmas 54169

[13] Kruger S. 1999 Generalized Reduced Magnetohydrodynamic Equations University of Wisconsin Center for Plasma Theory and Computation Report UW-CPTC 99-2

[14] Hegna C.C. and Callen J.D. 1994 Phys. Plasmas 12308

[15] Glasser A., Greene J. and Johnson J. 1975 Phys. Fluids 18875

[16] Connor J.W. et al 1988 Phys. Fluids 311539

[17] Dewar R.L. and Persson M. 1993 Phys. Fluids B 54274

[18] Fitzpatrick R. 1995 Phys. Plasmas 2825

[19] Sauter O. et al 1997 Phys. Plasmas 41654

[20] Reimerdes H., Sauter O., Goodman T. and Pochelon A. 2002 Phys. Rev. Lett. 88105005

[21] Smolyakov A.I., Hirose A., Lazzaro E., Re G.B. and Callen J.D. 1995 Phys. Plasmas 21581

[22] Lütjens H., Luciani J.-F. and Garbet X. 2001 Phys. Plasmas 84267

[23] Gianakon T.A., Hegna C.C. and Callen J.D. 1996 Phys. Plasmas 54637 